Document downloaded from:

http://hdl.handle.net/10251/103300

This paper must be cited as:

Barrera Vilar, D.; Madrigal-Madrigal, J.; Sales Maicas, S. (2017). Tilted fiber Bragg gratings in multicore optical fibers for optical sensing. Optics Letters. 42(7):1460-1463. doi:10.1364/OL.42.001460

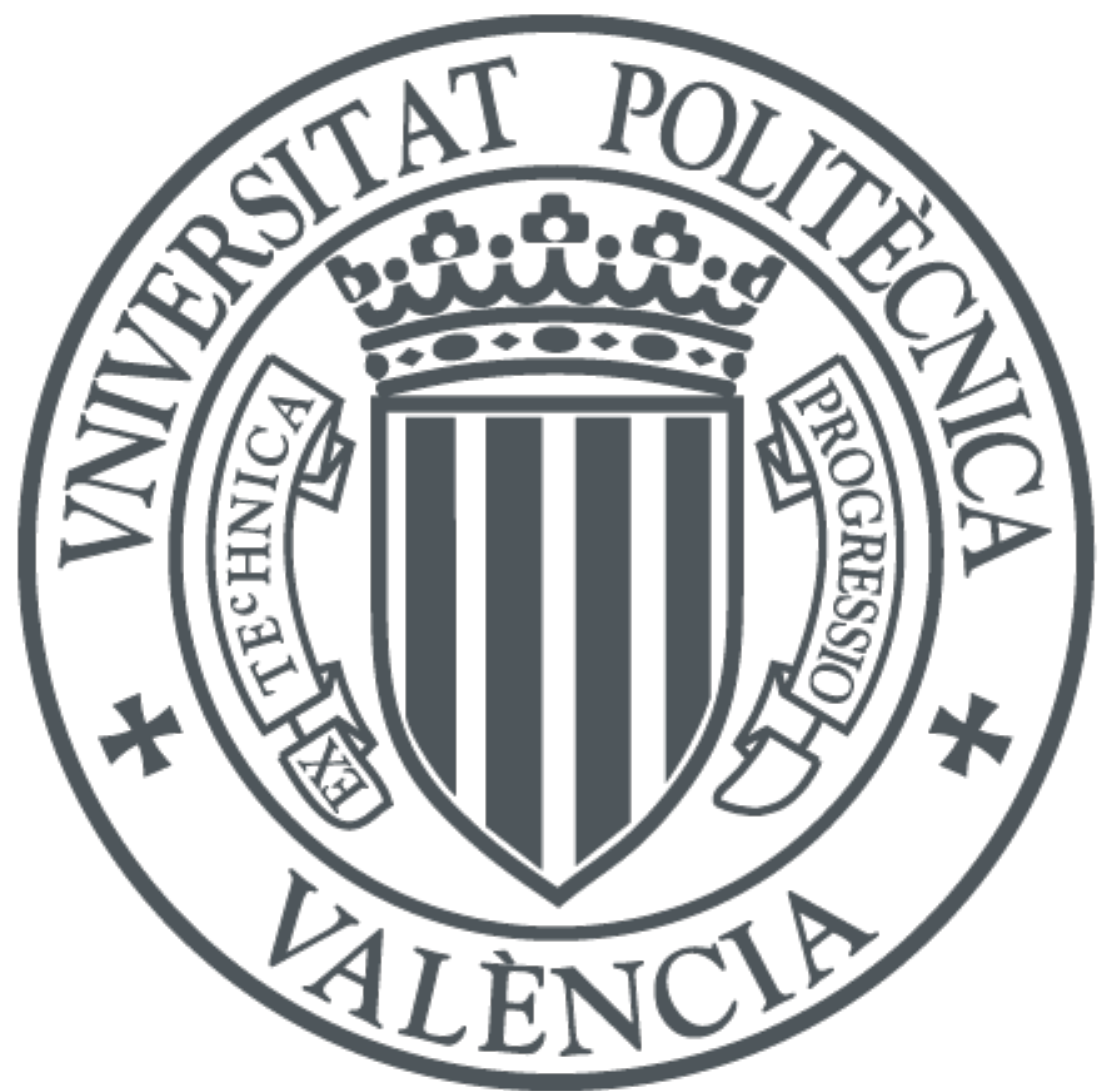

The final publication is available at

http://doi.org/10.1364/OL.42.001460

Copyright The Optical Society

Additional Information 


\title{
Tilted fiber Bragg gratings in multicore optical fibers for optical sensing
}

\author{
DAVID BARRERA, * JAVIER MADRIGAL AND SALVADOR SALES
}

\author{
${ }^{1}$ Photonics Research Labs, ITEAM research institute, Universitat Politècnica de València, Camino de Vera, s/n, 46022, Valencia, Spain \\ *Corresponding author: dabarvi@iteam.upv.es
}

\begin{abstract}
We have inscribed a tilted fiber Bragg grating (TFBG) in selected cores of a multicore optical fiber. The presence of the TFBG permits to couple light from the incident guided mode to the cladding modes and to the neighbor cores and this interaction can be used for optical sensing. We have considered to measure different magnitudes: strain, curvature magnitude and direction and external refractive index. The curvature results show a linear dependence of the maximum crosstalk with the curvature magnitude with a sensitivity of $2.5 \mathrm{~dB} / \mathrm{m}^{-1}$ as the curvature magnitude increases and at the same time a wavelength shift of $70 \mathrm{pm} / \mathrm{m}^{-1}$. Changes in the external refractive index gradually vanish the cladding modes resonances and the crosstalk between the different cores, obtaining a reduction of the $90 \%$ of the optical spectra integral area for refractive indexes between 1.398 to 1.474. (C) 2016 Optical Society of America
\end{abstract}

OCIS codes: (060.3735) Fiber Bragg gratings; (060.2370) Fiber optics sensors.

http://dx.doi.org/10.1364/OL.99.099999

Fiber optic sensors have attracted a considerable interest due to their intrinsic properties over conventional sensors like immunity to electromagnetic interferences, low attenuation, negligible ignition risk, chemically inert, hard environmental conditions resistant and sometimes the possibility of multiplexing the sensors in the same optical fiber. The intense research in this area has led to the fabrication of numerous devices, used to measure a wide range of magnitudes for various applications $[1,2]$.

In the last years, the development of multicore optical fibers (MCF) and their use for optical sensing applications has demonstrated the possibility to perform complex measurements, however, the potential of MCFs has not been fully explored. Previous research is mainly focused in physical parameters like curvature magnitude and direction in 3D shape sensing or 3D accelerometer [3-6]. New devices and approaches are needed in order to take advantage of the special properties of MCFs for optical sensing. MCFs are generally designed for telecommunication applications and they have very low crosstalk between different cores and consequently a very low interaction between the cores and the external medium. For numerous sensing applications, it is essential to enhance the influence of the external medium and different approaches have been proposed for this purpose in singlecore optical fibers: the partial or complete etching of the fiber cladding; the use of tapers or specialty fibers; and the inscription of optical gratings [7-15]. The use of tilted fiber Bragg grating (TFBG) permits to couple part of the light from the incident guided mode to the cladding modes that are more sensitive to external changes without altering the physical properties of the optical fiber. A TFBG in MCF also permits to the light propagating in one of the cores to be coupled to any of the other cores. These properties make this device a very interesting study subject for sensing applications. To the best of our knowledge, in this paper we show for the first time the selective inscription of TFBGs in a MCF and we describe the interaction between cores under external perturbations.

The MCF used is a seven-core optical fiber from Fibercore Ltd. with a cladding diameter of $125 \mu \mathrm{m}$. The core arrangement follows a hexagonal core pattern plus a central core with a separation between cores or pitch of $35 \mu \mathrm{m}$. Each core has a mode field diameter of $6.4 \mu \mathrm{m}$ and a numerical aperture of 0.2 .

Before the grating inscription, the photosensitivity of the fiber was increased with a hydrogen loading process at 50 bar for at least 15 days at ambient temperature. Two fan in/out devices provided by Optoscribe Ltd. were spliced to both ends of the MCF in order to access the individual cores during the inscription and the later characterization. The TFBG has been inscribed using the phasemask method. We have employed a CW frequency-doubled Argonion laser emitting at $244 \mathrm{~nm}$. The inscription setup has been developed for optimizing the irradiation of the laser beam into individual cores of the optical fiber [16]. The core tracking subsystem is based on the photoluminescence generated by the UV radiation $[17,18]$ and is essential when using a very narrow laser beam and MCFs, since it provides the spatial position of the different cores within the cross-sectional area of the optical fiber. Our setup can be used to inscribe simultaneously in all the cores, in a group of cores or just in a specific core controlling the laser beam dimensions and having a precise knowledge of the spatial position of each core inside the MCF. In order to inscribe a TFBG the phase mask is rotated $3^{\circ}$ along the laser beam axis. Figure 1 (a) shows a lateral and a frontal schematic view of the relative position of the cores inside the MCF and the phase mask while the TFBG is inscribed. A TFBG has been simultaneously inscribed in three of the cores of the MCF 
in order to study the interaction between the different cores with and without TFBGs. The picture at the bottom of Fig. 1(a) describes the interaction between the incident guided mode and the modes guided in the neighbor cores. We use the terms crosstalk (XT) for the light coupled to any other core in the same direction of the guided mode and back crosstalk (BXT) for the light coupled to any other core in the opposite direction. To denote the XT or BXT
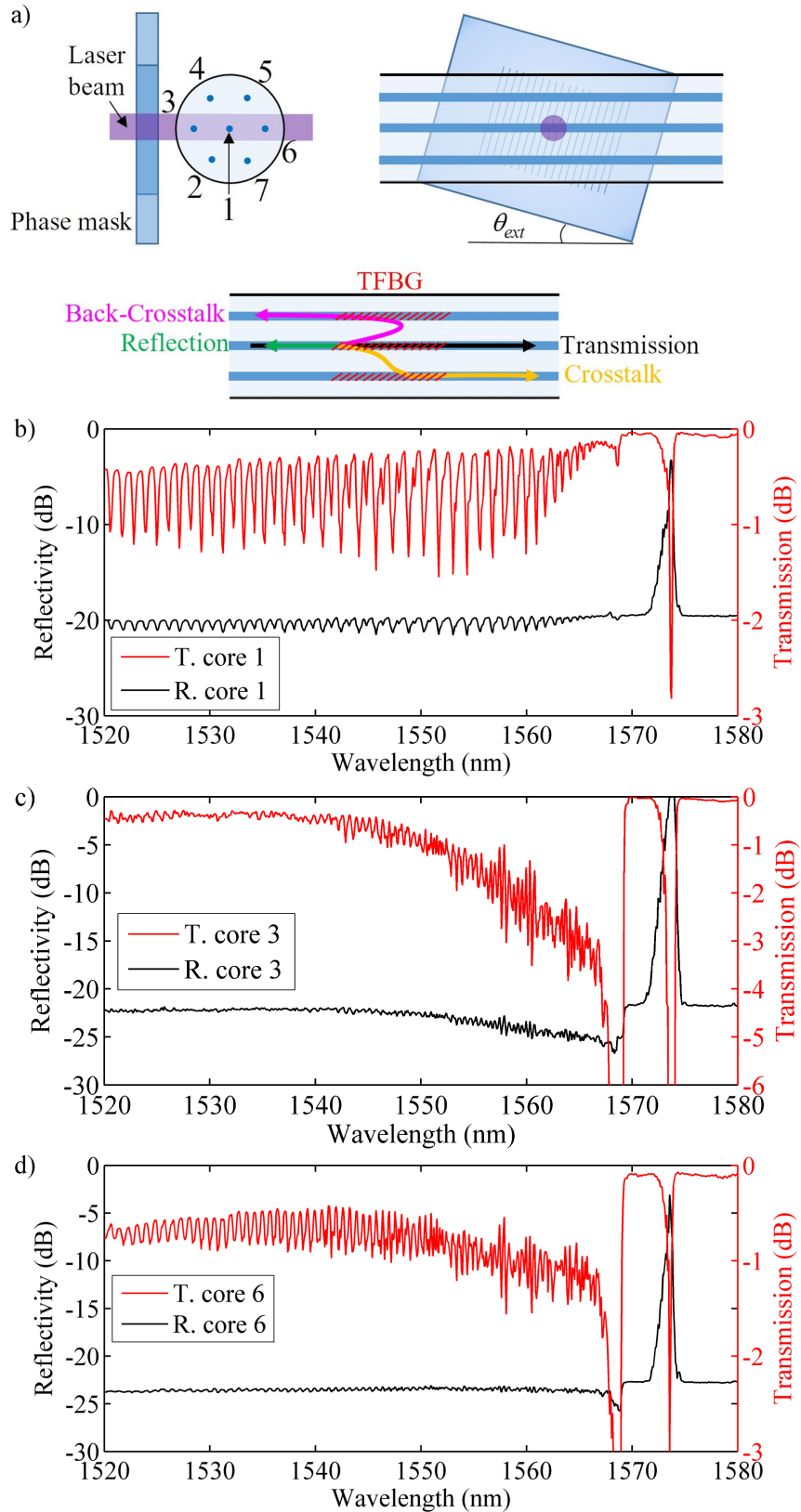

Fig. 1. a) Schematic view of the MCF and the phase mask while the TFBG is inscribed and interaction between cores. Reflected and transmitted optical spectra. b) Core 1. c) Core 3. d) Core 6.

between specific cores we use a right arrow $(\rightarrow)$ between the core numbers to indicate the core where the incident mode is propagating and the core where the measurement is performed.
All the measurements were obtained using an optical spectrum analyzer ANDO AQ6317C synchronized with a tunable laser source AND0 AQ4321D. Figures 1(b) to 1(d) shows the reflected and the transmitted optical spectra of the $5 \mathrm{~mm}$ long TFBG inscribed in cores 1, 3 and 6, respectively. These figures reveal significant differences between the spectra of the TFBGs in the outer cores and in the center core. There are a higher number of cladding modes and the wavelength spacing between them is shorter than in the outer cores due to their proximity to the external medium.
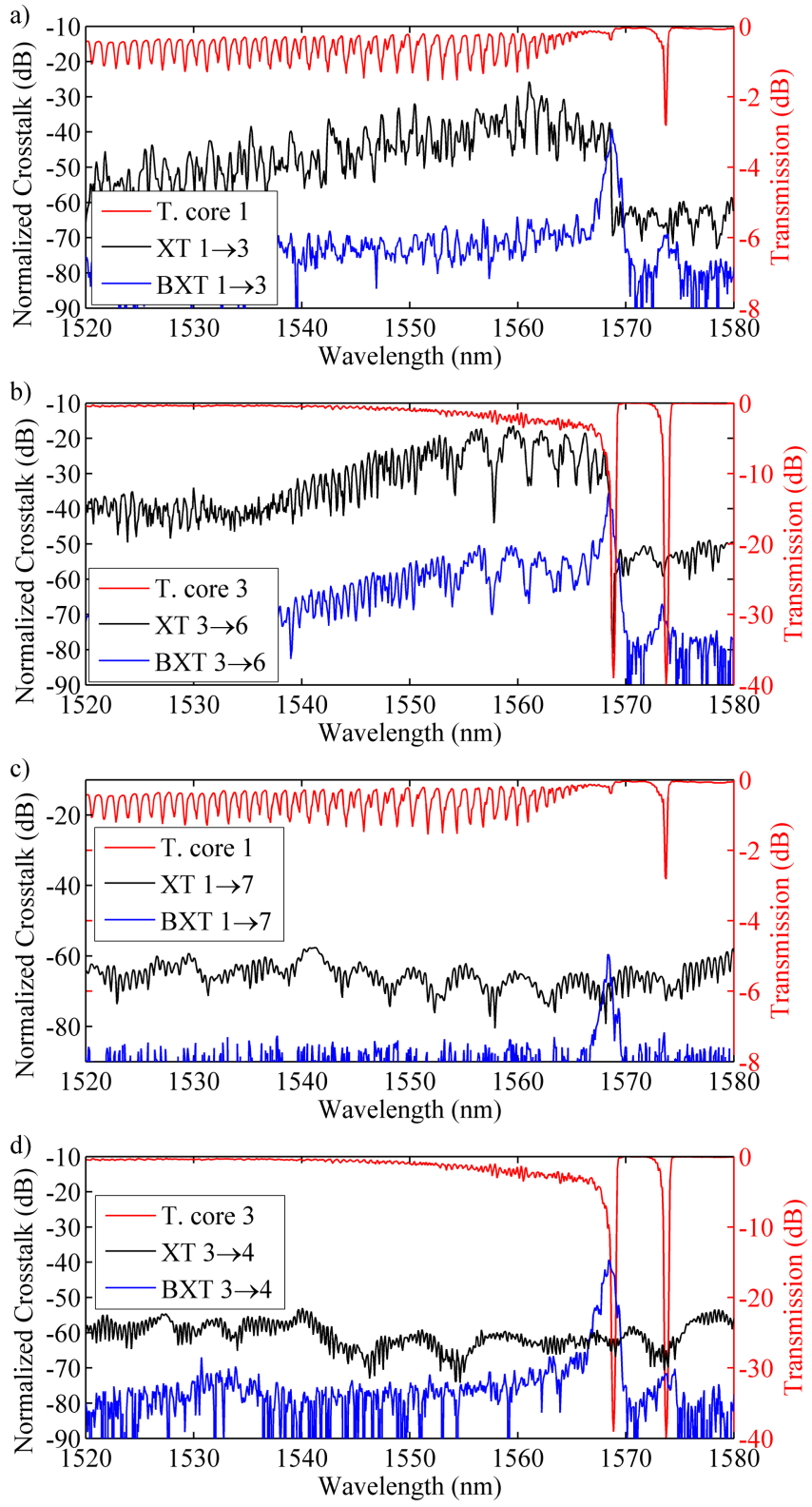

Fig. 2. Transmission spectra of inscribed TFBG, crosstalk and backcrosstalk. a) Between cores 1 and 3. (b) Between cores 3 and 6 . c) Between cores 1 and 7. d) Between cores 3 and 4.

Figure 2 shows the interaction between different pairs of cores. The crosstalk level measured by the optical spectrum analyzer is normalized to the optical power of the incident guided mode. Figure 2(a) exposes the normalized XT and BXT between core 1 and 
core 3 (XT $1 \rightarrow 3$ and BXT $1 \rightarrow 3$ ). As expected, the attenuation bands in the transmission spectra match the local maximums in the XT and BXT spectra. Additionally, the results reveal that the ghost mode is coupled to the outer cores in both directions while the high-order cladding modes are stronger coupled in the same direction of the guided mode. The same results have been obtained between core 1 and core 6 . Figure 2(b) confirms a different interaction between the two external cores. The XT $3 \rightarrow 6$ shows fewer cladding modes and a bigger wavelength spacing near the ghost mode. This effect is produced by the non-symmetric effective refractive index around the outer cores due to the proximity of the external medium and is consistent with the results previously reported about the effects of cladding etching in TFBGs inscribed in single-core fibers $[11,12]$. It can be observed that the BXT $3 \rightarrow 6$ is slightly higher than the BXT $1 \rightarrow 3$. The interaction between cores 1 , 3 and 6 and the other cores in the MCF have been also studied. Figure 2(c) and 2(d) show two examples of this interaction, between cores 1 and 7 and cores 3 and 4 . The XT is normally below $-50 \mathrm{~dB}$, that is close to the crosstalk specification for the fan in/out devices used, while the BXT shows a resonance in the ghost mode wavelength. Similar results are obtained for other pairs of cores.

To demonstrate the properties of the TFBGs in MCFs for sensing applications we have considered to measure different magnitudes: strain, curvature magnitude and direction and external refractive index.

Figure 3(a) shows the setup used to test the performance of the proposed device. First, we secured the MCF between one of the translation stages and the fixed platform for measuring the effect of the strain. We observed the transmission spectra of each core and theXT and BXT spectra between pairs of cores while the strain was applied. Figure 3(b) shows the wavelength shift for the resonances of the central core. The results revealed a sensitivity of $1.187 \mathrm{pm} / \mu \varepsilon$ at the Bragg wavelength while the sensitivities of the cladding modes located at different wavelengths are lower. The sensitivities are displayed inside figure 3(b). The lower the wavelength of the cladding mode, the lower the sensitivity. Cores 3 and 6 show similar results, as expected. The strain can also be measured using the XT or the BXT, which adds versatility to compensate cross effects and to implement novel configurations. The ghost mode showed in BXT $1 \rightarrow 3$ has a strain sensitivity of $1.17 \mathrm{pm} / \mu \varepsilon$, which is different from the sensitivity of the Bragg wavelength of the TFBG.

A uniform metal strip was situated between one of the translation stages and the fixed platform to measure the curvature magnitude and direction. The second translation stage was used to minimize any additional strain in the optical fiber while the metal strip was curved. The rotation stages allowed to change the curvature direction. Initially, cores 1, 3 and 6 were vertically aligned with core 3 in the top position. The curvature was progressively increased from $0 \mathrm{~m}^{-1}$ to $4 \mathrm{~m}^{-1}$ taking care not to apply any additional strain. The test was then repeated with a $90^{\circ}$ rotation in the MCF. Figure 3(c) illustrates the results obtained for the XT between cores 1,3 and 6 . When the three cores are vertically aligned (filled markers), the maximum of the XT $1 \rightarrow 3$ and the XT $3 \rightarrow 6$ decrease as the curvature is increased at a rate of approximately of $2.5 \mathrm{~dB} / \mathrm{m}^{-1} \mathrm{in}$ both cases. In contrast, the XT $1 \rightarrow 6$ remains almost constant.
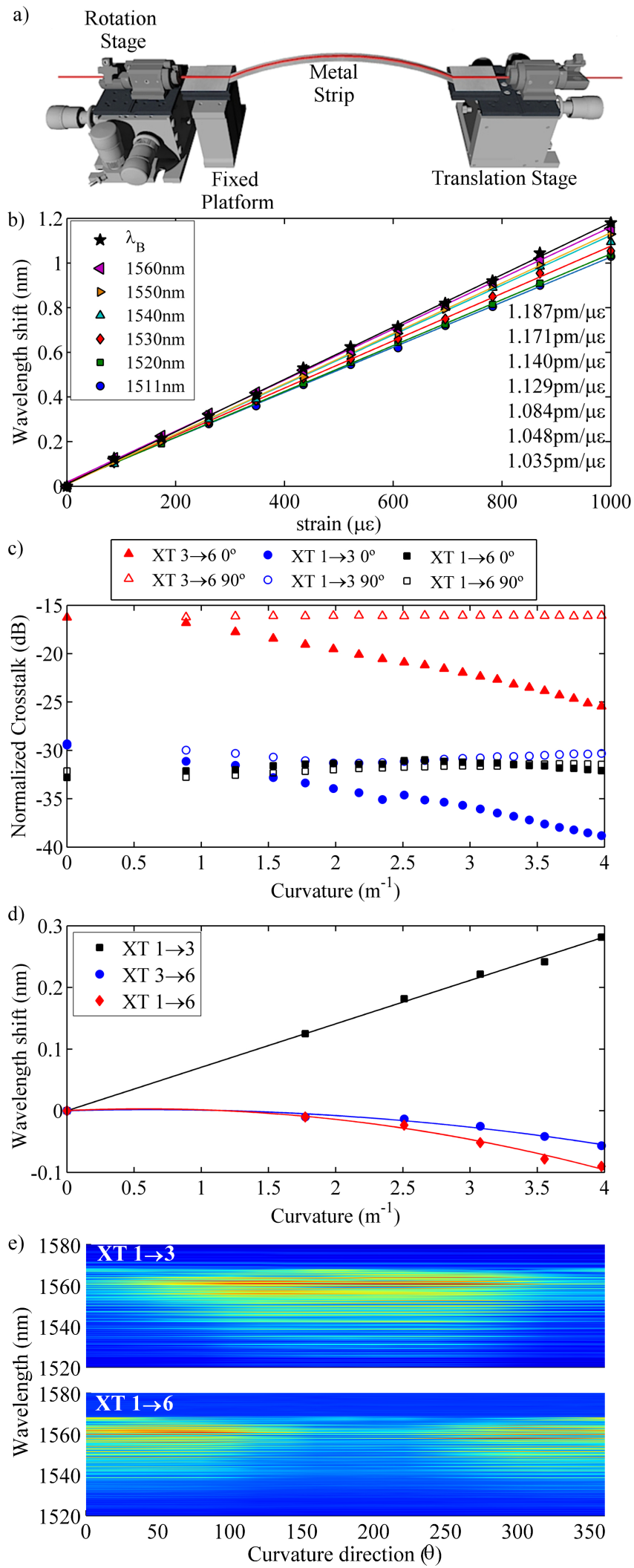

Fig. 3. a) Setup used for experimental measurements. b) Strain sensitivity of different cladding modes in core 1. c) Maximum XT between cores 1, 3 and 6 with curvature magnitude. d) Spectra shift of the XT between cores 1, 3 and 6 with curvature magnitude. e) XT $1 \rightarrow 3$ and XT $1 \rightarrow 6$ evolution with curvature direction. 
When the MCF is rotated $90^{\circ}$ (unfilled markers), the XT $1 \rightarrow 3$ and XT $1 \rightarrow 6$ shows a similar constant level. The differences observed in the behavior of XT $1 \rightarrow 6$ are a consequence of the relative position of the core 6 inside the MCF and the cladding modal intensity distribution when the fiber is bent. Additionally, the XT and the BXT spectra exhibits a wavelength shift when the curvature is applied, see Fig. 3(d). The wavelength shift observed for the XT $1 \rightarrow 3$ measured near the ghost mode is approximately linear with a sensitivity of $70 \mathrm{pm} / \mathrm{m}^{-1}$. The wavelength shift observed for the XT $1 \rightarrow 6$ and XT $3 \rightarrow 6$ is negative as consequence of the relative position of the cores inside the optical fiber and the experimental results are best fitted by a second order polynomial. We applied a constant curvature of $4 \mathrm{~m}^{-1}$ and then we used the rotation stages to change the curvature direction in steps of $20^{\circ}$ to observe the curvature direction dependency. Figure 3(e) shows the evolution of the XT $1 \rightarrow 3$ and XT $1 \rightarrow 6$ spectra with the curvature direction where it is evident the complementary behavior of the crosstalk.

The external refractive index (RI) was also measured using the TFBGs of the MCF. The MCF was maintained suspended and straight between the two translation stages. A container is then placed just below without any contact with the TFBGs. Glycerol dissolutions $(50,60,70,85$ and $100 \mathrm{wt} \%)$ was used in order to get external refractive indexes from 1.398 to 1.474 . Figure 4 (a) shows theXT $3 \rightarrow 6$ spectra for increasing RIs. The increment of the effective refractive index causes a gradual vanishing of the cladding modes. Figure 4(b) shows the integral area of the XT spectra normalized to the value in air. It can be observed a similar behavior of the XT between cores 1, 3 and 6 . A reduction of almost $90 \%$ of the integral area is observed for the studied RI range.
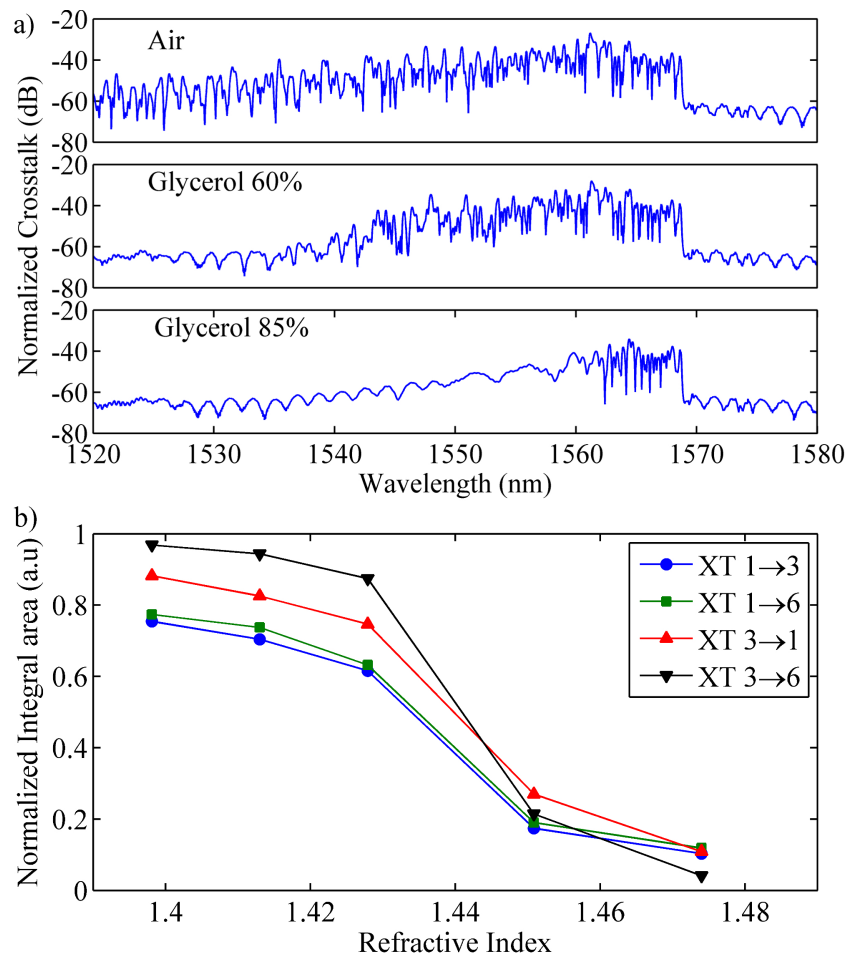

Fig. 4. a) XT $3 \rightarrow 6$ spectra under different external refractive indexes. b) Evolution in the normalized integral area of the XT spectra with the external refractive index change.
In summary, we have selectively inscribed, for the first time, TFBGs in a group of cores of a MCF and we have studied the interaction between the different cores. Important differences were found between the transmission spectra of the TFBG inscribed in the outer cores and in the central core. We have analyzed the crosstalk between different cores produced by the presence of the TFBG and we have investigated the dependence of the crosstalk under external perturbations, focusing in particular on the effects of strain, curvature magnitude and direction and external refractive index. The new device shows a strong dependence of the maximum crosstalk from both the curvature magnitude and the position of the outer core. When the cores are aligned with the curvature direction, the crosstalk is reduced at a rate of $2.5 \mathrm{~dB} / \mathrm{m}^{-1}$ as the curvature magnitude increases. When the cores are perpendicular to the curvature direction the curvature has a minimum effect on the crosstalk. Curvature also produces a wavelength shift that can be measured in theXT, the sensitivity obtained for only one of the outer cores is $70 \mathrm{pm} / \mathrm{m}^{-1}$. Finally, the effect of external refractive index has been analyzed observing the gradually vanishing of the cladding modes resonances and a similar reduction of the XT between the different cores.

Funding. Research Award Program (GVA PROMETEO 2013/012); Spanish Ministry of Economy and Competitiveness (TEC201460378-C2-1-R); Generalitat Valenciana (APOSTD/2016/015).

\section{References}

1. G. Rajan, ed., Optical Fiber Sensors: Advanced Techniques and Applications (CRC Press, 2015).

2. J. L. Santos, and F. Farahi, eds., Handbook of Optical Sensors (CRC Press, 2015).

3. G.M.H. Flockhart, W.N. MacPherson, J.S. Barton, J.D.C. Jones, L. Zhang, and I. Bennion, Opt. Lett. 28, 387 (2003).

4. A. Fender, W.N. MacPherson, R.R.J. Maier, J.S. Barton, D.S. George, R.I. Howden, G.W. Smith, B.J.S. Jones, S. McCulloch, X. Chen, R. Suo, L. Zhang, and I. Bennion, IEEE Sens. J. 8, 1292 (2008).

5. C. Askins, T. Taunay, G. Miller, B. Wright, J. Peele, L. Wasserman, and E. Friebele, in Bragg Gratings, Photosensitivity, and Poling in Glass Waveguides, OSA Technical Digest (CD) (Optical Society of America, 2007), paper JWA39.

6. D. Barrera, I. Gasulla, and S. Sales, J. Lightw. Technol. 33, 2445 (2015).

7. C.A.J. Gouveia, J.M. Baptista, and P.A.S. Jorge, Current Developments in Optical Fiber Technology (InTech, 2013).

8. J.M. Corres, F.J. Arregui, and I.R. Matías, Sensor Actuat. B-Chem. 122, 442 (2007).

9. D. Barrera, J. Villatoro, V.P. Finazzi, G.A. Cárdenas-Sevilla, V.P. Minkovich, S. Sales, and V. Pruneri, J. Lightw. Technol. 28, 3542 (2010).

10. P. Kisała, D. Harasim, and J. Mroczka, Opt. Express 24, 29922 (2016)

11. J. Albert, L.-Y. Shao, and C. Caucheteur, Laser Photon. Rev. 7, 83 (2012).

12. C. Chen, C. Caucheteur, P. Mégret, and J. Albert, Meas. Sci. Technol. 18, 3117 (2007).

13. X. Dong, H. Zhang, B. Liu, and Y. Miao, Photonic Sensors 1,6 (2010).

14. T. Guo, F. Liu, B.-O. Guan, and J. Albert, Opt. Laser Technol. 78, 19 (2016).

15. Z. Cai, F. Liu, T. Guo, B.-O. Guan, G.-D. Peng, and J. Albert, Opt. Express 23, 20971 (2015).

16. I. Gasulla, D. Barrera, J. Hervás, and S. Sales, Sci. Rep. (To be published). 17. M. Gallagher, and U. Österberg, Appl. Phys. Lett. 63, 2987 (1993). 18. T. Komukai and M. Nakazawa, IEEE Photon. Tech. Lett. 8, 1495 (1996). 
1. G. Rajan, ed., Optical Fiber Sensors: Advanced Techniques and Applications (CRC Press, 2015).

2. J. L. Santos, and F. Farahi, eds., Handbook of Optical Sensors (CRC Press, 2015).

3. G.M.H. Flockhart, W.N. MacPherson, J.S. Barton, J.D.C. Jones, L. Zhang, and I. Bennion, "Two-axis bend measurement with Bragg gratings in multicore optical fiber," Opt. Lett. 28, 387-389 (2003).

4. A. Fender, W.N. MacPherson, R.R.J. Maier, J.S. Barton, D.S. George, R.I. Howden, G.W. Smith, B.J.S. Jones, S. McCulloch, X. Chen, R. Suo, L. Zhang, and I. Bennion, "Two-axis temperature-insensitive accelerometer based on multicore fiber Bragg gratings," IEEE Sens. J. 8, 1292-1298 (2008).

5. C. Askins, T. Taunay, G. Miller, B. Wright, J. Peele, L. Wasserman, and E. Friebele, "Inscription of Fiber Bragg Gratings in Multicore Fiber," in Bragg Gratings, Photosensitivity, and Poling in Glass Waveguides, OSA Technical Digest (CD) (Optical Society of America, 2007), paper JWA39.

6. D. Barrera, I. Gasulla, and S. Sales, "Multipoint two-dimensional curvature optical fiber sensor based on a non-twisted homogeneous four-core fiber," J. Lightw. Technol. 33, 2445-2450 (2015).

7. C.A.J. Gouveia, J.M. Baptista, and P.A.S. Jorge, Current Developments in Optical Fiber Technology (InTech, 2013), Chap. 13.

8. J.M. Corres, F.J. Arregui, and I.R. Matías, "Sensitivity optimization of tapered optical fiber humidity sensors by means of tuning the thickness of nanostructured sensitive coatings," Sensor Actuat. B-Chem. 122, 442-449 (2007).

9. D. Barrera, J. Villatoro, V.P. Finazzi, G.A. Cárdenas-Sevilla, V.P. Minkovich, S. Sales, and V. Pruneri, "Low Loss Photonic Crystal Fiber Interferometers for Sensor Networks," J. Lightw. Technol. 28, 35423547 (2010).

10. P. Kisała, D. Harasim, and J. Mroczka, "Temperature-insensitive simultaneous rotation and displacement (bending) sensor based on tilted fiber Bragg grating," Opt. Express 24, 29922-29929 (2016).

11. J. Albert, L.-Y. Shao, and C. Caucheteur, "Tilted fiber Bragg grating sensors," Laser Photon. Rev. 7, 83-108 (2012).

12. C. Chen, C. Caucheteur, P. Mégret, and J. Albert, "The sensitivity characteristics of tilted fibre Bragg grating sensors with different cladding thicknesses," Meas. Sci. Technol. 18, 3117-3122 (2007).

13. X. Dong, H. Zhang, B. Liu, and Y. Miao, "Tilted fiber Bragg gratings: Principle and sensing applications," Photonic Sensors 1, 6-30 (2010).

14. T. Guo, F. Liu, B.-O. Guan, and J. Albert, "Tilted fiber grating mechanical and biochemical sensors," Opt. Laser Technol. 78, 19-33 (2016).

15. Z. Cai, F. Liu, T. Guo, B.-O. Guan, G.-D. Peng, and J. Albert, "Evanescently coupled optical fiber refractometer based a tilted fiber Bragg grating and a D-shaped fiber," Opt. Express 23, 20971-20976 (2015).

16. I. Gasulla, D. Barrera, J. Hervás, and S. Sales "Spatial Division Multiplexed Microwave Signal processing by selective grating inscription in homogeneous multicore fibers," Sci. Rep. (To be published).

17. M. Gallagher, and U. Österberg, "Time resolved $3.10 \mathrm{eV}$ luminescence in germanium-doped silica glass," Appl. Phys. Lett. 63, 2987-2988 (1993).

18. T. Komukai and M. Nakazawa, "Fabrication of high-quality long fiber Bragg grating by monitoring $3.1 \mathrm{eV}$ radiation $(400 \mathrm{~nm})$ from GeO defects," IEEE Photon. Tech. Lett. 8, 1495-1497 (1996). 